\title{
High-entropy alloys as high-temperature thermoelectric materials
}

\author{
Samrand Shafeie, ${ }^{1,2}$ Sheng Guo, ${ }^{1, a)}$ Qiang Hu, ${ }^{3}$ Henrik Fahlquist, ${ }^{4}$ Paul Erhart, ${ }^{5}$ \\ and Anders Palmqvist ${ }^{2, b)}$ \\ ${ }^{1}$ Surface and Microstructure Engineering Group, Materials and Manufacturing Technology, \\ Chalmers University of Technology, SE-41296 Gothenburg, Sweden \\ ${ }^{2}$ Department of Chemistry and Chemical Engineering, Chalmers University of Technology, \\ SE-41296 Gothenburg, Sweden \\ ${ }^{3}$ Institute of Applied Physics, Jiangxi Academy of Sciences, Nanchang 330029, People's Republic of China \\ ${ }^{4}$ Bruker AXS Nordic AB, 17067 Solna, Sweden \\ ${ }^{5}$ Department of Applied Physics, Chalmers University of Technology, SE-41296 Gothenburg, Sweden
}

(Received 27 August 2015; accepted 26 October 2015; published online 12 November 2015)

\begin{abstract}
Thermoelectric (TE) generators that efficiently recycle a large portion of waste heat will be an important complementary energy technology in the future. While many efficient TE materials exist in the lower temperature region, few are efficient at high temperatures. Here, we present the high temperature properties of high-entropy alloys (HEAs), as a potential new class of high temperature TE materials. We show that their TE properties can be controlled significantly by changing the valence electron concentration (VEC) of the system with appropriate substitutional elements. Both the electrical and thermal transport properties in this system were found to decrease with a lower VEC number. Overall, the large microstructural complexity and lower average VEC in these types of alloys can potentially be used to lower both the total and the lattice thermal conductivity. These findings highlight the possibility to exploit HEAs as a new class of future high temperature TE materials. C 2015 AIP Publishing LLC. [http://dx.doi.org/10.1063/1.4935489]
\end{abstract}

\section{INTRODUCTION}

Waste heat recovery technologies are important future complements to renewable energy sources. ${ }^{1}$ During the last two decades, renewed interest in thermoelectric (TE) materials for efficient waste heat recovery has spawned research within nanostructured materials. ${ }^{2}$ In the search for TE materials with high conversion efficiency, the dimensionless TE figure-of-merit $z T$ has been used to estimate the performance. It is defined as

$$
z T=\frac{S^{2} \sigma}{k_{t o t}} T,
$$

where $S$ is the Seebeck coefficient, $\sigma$ is the electrical conductivity, $T$ is the absolute temperature in Kelvin, and $\kappa_{\text {tot }}$ is the total thermal conductivity. Maximizing the power factor ( $\left.\mathrm{PF}=S^{2} \sigma\right)$ while minimizing $\kappa_{\text {tot }}$ is the most widely employed strategy; however, due to the fundamentally interconnected nature of the three material parameters $(S, \sigma$, $\left.\kappa_{\tau o \tau}\right)$, the general approach for a specific material boils down to a strongly nonlinear optimization problem. ${ }^{1}$ To this end, new approaches related to low dimensional materials and nanostructuring for decoupling and changing the parameters independently have led to significant improvements in the $z T$ of current state-of-the-art TE materials. ${ }^{2}$

To reach industrially feasible materials for global use, high performance TE materials must contain low cost earth abundant elements with low toxicity. Yet, most high performing TE materials at low to medium temperatures ranging up to

\footnotetext{
a)E-mail: sheng.guo@chalmers.se

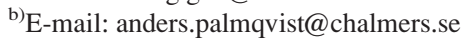

$\sim 600^{\circ} \mathrm{C}$ (e.g., $\mathrm{Bi}_{2} \mathrm{Te}_{3}, \mathrm{PbTe}$, and $\left.\left(\mathrm{Bi}_{1-\mathrm{x}} \mathrm{Sb}_{\mathrm{x}}\right)_{2}\left(\mathrm{Se}_{1-\mathrm{y}} \mathrm{Te}_{\mathrm{y}}\right)_{3}\right)$ contain toxic or scarce elements based on $p$-block elements, and recent focus has therefore shifted towards using new structure types (e.g., half-Heusler alloys ${ }^{3}$ ) to meet this prerequisite. For high temperature (HT) applications with $T>800^{\circ} \mathrm{C}$, the aforementioned materials based on $p$-block elements will quickly degrade, and therefore attention has been directed towards thermally more stable materials, e.g., oxides, magnesium silicides, and Zintl compounds. ${ }^{1,4,5}$ The advantages of HT TE materials are many (e.g., lower $z T$ is required to recover the same amount of energy compared with lower temperatures, and large scale industrial waste heat recovery is possible), though few materials at the moment deliver high performance $(z T>1)$ at $T>800^{\circ} \mathrm{C} .{ }^{1,2,6}$ Among the state-of-the-art metallic compounds and alloys with $n$-type conductivity, very few examples are known to exhibit high enough $z T$ values that can compete with the state-of-the-art $n$ type $\mathrm{SiGe}$ compounds ${ }^{1}$ at $T \geq 800^{\circ} \mathrm{C}$ with $z T \approx 1$ (e.g., halfHeusler type materials $\left.s^{3,7,8}\right)$. The main drawback of halfHeusler compounds have long been their large lattice $\left(\kappa_{\text {latt }}\right)$ and electrical thermal conductivity $\left(\kappa_{\mathrm{e}}\right)$ that hamper their use as commercial HT-TE materials $(z T$ at least $\sim 1$ at $\left.\sim 600-700^{\circ} \mathrm{C}\right) .{ }^{8}$ Some high performance half-Heusler compounds also contain costly precious metals such as $\mathrm{Pd},{ }^{9}$ or are only efficient at very low temperatures. ${ }^{10}$

Recently, high entropy alloys (HEAs) have been proposed as novel types of alloys with many intriguing structural and functional properties. ${ }^{11-13}$ HEAs are constituted of at least 5 different elements in equimolar or close to equimolar amounts, and the enhanced configurational entropic contribution, particularly at elevated temperatures, can thermodynamically stabilize the formation of solid solutions. ${ }^{14}$ Apart from the 
formation of solid solutions, the complex phase space of these alloys $^{15-17}$ can potentially be used as a means to achieve exceptional properties in areas where nanostructuring is of importance. $^{2,18,19}$ As a result, HEAs are currently being evaluated for, e.g., their mechanical properties. ${ }^{11,20-22}$ Many new and unexplored avenues still remain for these types of materials (e.g., superconductivity ${ }^{23}$ and soft magnetic materials ${ }^{24,25}$ ).

Generally, the reduction of $\kappa_{\text {latt }}$ is an important step towards a high $z T$ of a TE material. This usually requires the use of three key strategies: first, the scattering of phonons on atomic length scales through rattling atoms, vacancies, impurities, interstitials, or substitutional atoms (all related to point defects in the material); second, the concept of "phonon-glass electron crystal" (PGEC) ${ }^{26}$ should ideally be fulfilled, i.e., phonons are scattered by complexity or disorder in the crystal structure, while electrons move freely as in an "electron-crystal" (associated with the long range order in the material); and third, through interfaces, e.g., mesoscale grain and phase boundaries. $1,19,27$

In HEAs, all of the above phonon scattering strategies can in principle be achieved simultaneously through the intrinsically complex nature of the materials. In general, HEAs offer large amounts of complexity through severe lattice distortions, point defects, or the precipitation of secondary phases in order to scatter phonons effectively, while maintaining a high mobility of the conduction electrons. In addition to effective means for phonon scattering, HEAs possess mostly high symmetry crystal structures such as simple face centered cubic close packing (FCC) or body centered cubic close packing (BCC), or in some cases hexagonal cubic close packing (HCP). They are, therefore, also likely to achieve a high convergence of the bands close to the Fermi level to obtain high Seebeck coefficient values. $^{28}$ The challenge in achieving high $z T$ in these materials is at the moment, therefore, related to the decrease in the electrical conductivity that has a large negative impact on the total thermal conductivity, and also on the Seebeck coefficient due to the excessive number of charge carriers.

Controlling the properties with high accuracy in HEAs is a great challenge. However, some well-established rules for prediction of solid solutions in HEAs have been reported, ${ }^{29,30}$ and are based on the following parameters:

$$
\begin{gathered}
V E C=\sum_{i=1}^{n} c_{i}(V E C)_{i}, \\
\delta=\sqrt{\sum_{i=1}^{n} c_{i}\left(1-\frac{r_{i}}{\sum_{j=1}^{n} c_{j} r_{j}}\right)^{2}} \\
\Delta H_{m i x}=\sum_{i=1, j \neq i}^{n} 4 c_{i} c_{j} \Delta H_{A-B} .
\end{gathered}
$$

Here, the valence electron concentration (VEC) is the total number of valence electrons including $d$-electrons, $\delta$ is the weighted atomic radii mismatch, and $\Delta H_{\text {mix }}$ is the total weighted $\Delta H_{A-B}$ (based on Miedema's values for binary alloys $^{31}$ ). In the above equations, $c_{i}$ and $c_{j}$ are the atomic percentages of elements $i$ and $j$, and $r_{i}$ and $r_{j}$ are their atomic radii, respectively.

To our knowledge, HEAs have not been reported before in the context of $\mathrm{TE}$, and therefore, offer completely new possibilities regarding the exploration for HT-TE materials. ${ }^{32,33}$ Due to the difficulty of tuning the charge carrier concentration in metals, many alloys have not been considered for TE applications due to their large electron concentration and low Seebeck coefficients. Nevertheless, the possibility to form complex microstructures in HEAs (see, e.g., Refs. 16 and 17) offers opportunities for the reduction of the thermal conductivity by phonon scattering.

Here, we present the investigation of a model HEA system with the composition $\mathrm{Al}_{\mathrm{x}} \mathrm{CoCrFeNi}(0.0 \leq x \leq 3.0$, where $x$ is the atomic portion) as a potential HT-TE material. We show that for this system (for which only electrical and thermal conductivities at intermediate temperatures for $0.0 \leq x \leq 2.0$ have previously been reported ${ }^{32}$ ), the VEC (which is a well-established parameter for estimating the stability of FCC and BCC phase regions among HEA systems) can be used as a general parameter to change the electrical conductivity and Seebeck coefficients of these materials into suitable ranges for TE materials. As a result of the addition of $\mathrm{Al}$, which is inserted in order to decrease the VEC of the system, we observed a significant decrease in electrical conductivity. This decrease in electrical conductivity was followed by an increase in the absolute value of the Seebeck coefficient. Overall, we also observed a significant decrease in $\kappa_{\text {tot }}\left(=\kappa_{\mathrm{e}}+\kappa_{\text {latt }}\right)$, primarily due to a lower electrical contribution, $\kappa_{\mathrm{e}}$. Possible causes for the enhanced $z T$ are discussed mainly for compositions in the range of $2.0 \leq x \leq 3.0$.

\section{EXPERIMENTAL SECTION}

Ingots of $\mathrm{Al}_{x} \mathrm{CoCrFeNi}$ with $0.0 \leq x \leq 3.0$ and $\Delta x=0.25$ were prepared from commercially pure elements (purity $\geq 99.9$ wt. \%). The raw elements were alloyed by arcmelting in a Ti-gettered high-purity argon atmosphere. The melting of the ingots with intermediate flipping was repeated at least five times in order to achieve a good homogeneity of the alloys. The ingots were grinded and polished to obtain a smooth and clean surface. Phase constitutions of the ingots were obtained with a Bruker x-ray diffractometer (XRD) D8 Advance diffractometer equipped with a $\mathrm{Cr}_{\alpha}$ target $\left(\lambda_{\alpha 1}=1.5406 \AA\right.$ and $\lambda_{\alpha 2}=1.54439 \AA$ ), operated at a voltage of $35 \mathrm{kV}$ and a current of $40 \mathrm{~mA}$. Data were acquired in the $2 \theta$ range of $10^{\circ}-135^{\circ}$ with a step size of $0.08^{\circ} /$ step and $6 \mathrm{~s} /$ step. To observe if additional phases were formed, samples with $x=0.25,0.75,1.25$, and 3.0 were analyzed by XRD after the electrical transport measurements. Differential scanning calorimetry (DSC) measurements were performed on small pieces of as-cast samples $(\sim 40-50 \mathrm{mg})$ placed in an $\mathrm{Al}_{2} \mathrm{O}_{3}$ crucible and heated in a Netzsch STA 449F3. Heating and cooling were performed in flowing Argon gas with a temperature ramp of $10^{\circ} \mathrm{C} \min ^{-1}$ from 30 to $900{ }^{\circ} \mathrm{C}$. The high temperature thermal conductivity, $\kappa_{\text {tot }}$, of the ingots $(x=0.0,2.0,2.25,2.5,2.75$, and 3.0) was measured in a Hot Disk Thermal Constant Analyser TPS 2500S. The data were analyzed by using the Hot Disk Thermal Constant Analyser (Version 7.1.22) software. A Hot Disk sensor C5465 with a radius of $3.189 \mathrm{~mm}$ and a double spiral made of $\mathrm{Ni}$ were used for the measurements. Due to the Curie transition $\left(355^{\circ} \mathrm{C}\right)$ of the Nickel sensor, data were not measured 
between 300 and $450{ }^{\circ} \mathrm{C}$ in order to obtain physically sensible results. Measurements were made in helium atmosphere by means of the transient plane source (TPS) method $^{34}$ between 105 and $505^{\circ} \mathrm{C}$ with 4 measurements at each temperature to obtain better accuracy. The presented results are averages of the 4 measurement points, where the corresponding standard deviation at each temperature is indicated with an error bar in the figures. For all samples, a measurement time of $2 \mathrm{~s}$ with an applied power output between 150 and $250 \mathrm{~mW}$ was used with a waiting time between 40 and $60 \mathrm{~min}$ between each measurement. Single sided measurements were made with the sensor sandwiched between the flat polished ingot sample surface and thermally insulating quartz fiber. The error for the measurement points is estimated to be $\sim 3 \%-5 \%$ on average among measured points at each temperature.

Temperature dependent electrical resistivity $(1 / \sigma)$ and Seebeck coefficients $(S)$ for different samples were measured using as-cast samples in an ULVAC ZEM-3 instrument. Sample dimensions ranged between $\sim 6$ and $11 \mathrm{~mm}$ in length, with an average side length/diameter of $\sim 2-3 \mathrm{~mm}$. Measurements were performed from room temperature to $\sim 900^{\circ} \mathrm{C}$ with $\sim 0.1$ bar helium gas in the measurement chamber. In the ULVAC ZEM-3, the resistivity $(1 / \sigma)$ was measured with a standard 4-point probe method by sending the current through the sample rod, while simultaneously measuring the voltage difference along the length of the rod. The Seebeck coefficient was simultaneously obtained by heating one end of the sample, while measuring the generated voltage between the probes. For each measured temperature point of the Seebeck coefficient, temperature differences of $\Delta T=20,30$, and $40^{\circ} \mathrm{C}$ were used in order to minimize measurement errors. All measurements were performed using heating and cooling cycles in order to observe possible hysteresis effects in the as-cast samples. The hysteresis effect from the first heating in the samples has been excluded due to irreversible changes when starting from an as-cast state; the presented values are thus represented as average values for the measured temperature ranges with an estimated total error of $\leq \sim 3 \%$ between two consecutive measurements. For comparison, remeasured values for $x=0.0$ and 1.75 are shown in the supplementary material for the electrical conductivity (Figure S1, supplementary material), Seebeck coefficients (Figure S2, supplementary material), and power factors (Figure S3, supplementary material); in addition, the thermal conductivity (Figure S4, supplementary material) for $x=2.25$ was remeasured after remelting to check the reproducibility of the thermal conductivity values for the same sample. ${ }^{35}$ In addition, the total, electronic, and lattice thermal conductivities are also included for $x=2.0$, $2.25,2.5,2.75$, and 3.0 as additional information (Figures S5-S9, supplementary material). ${ }^{35}$ Overall, it should be noted that the 1 st heating from room temperature up to $900{ }^{\circ} \mathrm{C}$ is excluded, due to a hysteresis effect in the electrical conductivity and Seebeck coefficients that can be related to microstructural changes as well as local compositional changes. It was, however, noted that after reaching $900^{\circ} \mathrm{C}$ such effects were not observed in the 2 nd and 3 rd cycles. We observed that for some compositions with large $x>2$, the hysteresis could start already at $\sim 100^{\circ} \mathrm{C}$, thus indicating that equilibration might start already at those temperatures. The measurement time at $\sim 800-900{ }^{\circ} \mathrm{C}$ was estimated to be $5 \mathrm{~h}$ from the time stamps in the raw data, and the total time for 3 cycles at $T>500^{\circ} \mathrm{C}$ was $\sim 20 \mathrm{~h}$. Assuming that the equilibration starts already at $\sim 100^{\circ} \mathrm{C}$, equilibration has taken place for $\sim 90 \mathrm{~h}$ during the 3 cycles that were used. Hence, the average of those "equilibrated" measurements has been shown in this report. The microstructural effects of before and after annealing at the maximum temperature will be reported elsewhere and are not the focus of this investigation.

\section{RESULTS}

\section{Phase identification}

The XRD patterns of polished samples from the $\mathrm{Al}_{x} \mathrm{CoCrFeNi}$ ingots with $0.0 \leq x \leq 3.0$ are shown in Figure 1. It is observed that compositions with $x=0$ and 0.25 $(8.25 \geq$ VEC $\geq 7.94)$ appear to be close to pure FCC phase. Traces of secondary cubic phases are, however, observed in the XRD data as evident from the right shoulder at $\sim 80.6^{\circ}$ 20. Traces of tetragonal $\mathrm{Ni}_{1.2} \mathrm{Al}_{0.8}$ type intermetallic compounds are also observed at $\sim 41.35^{\circ} 2 \theta$. With larger

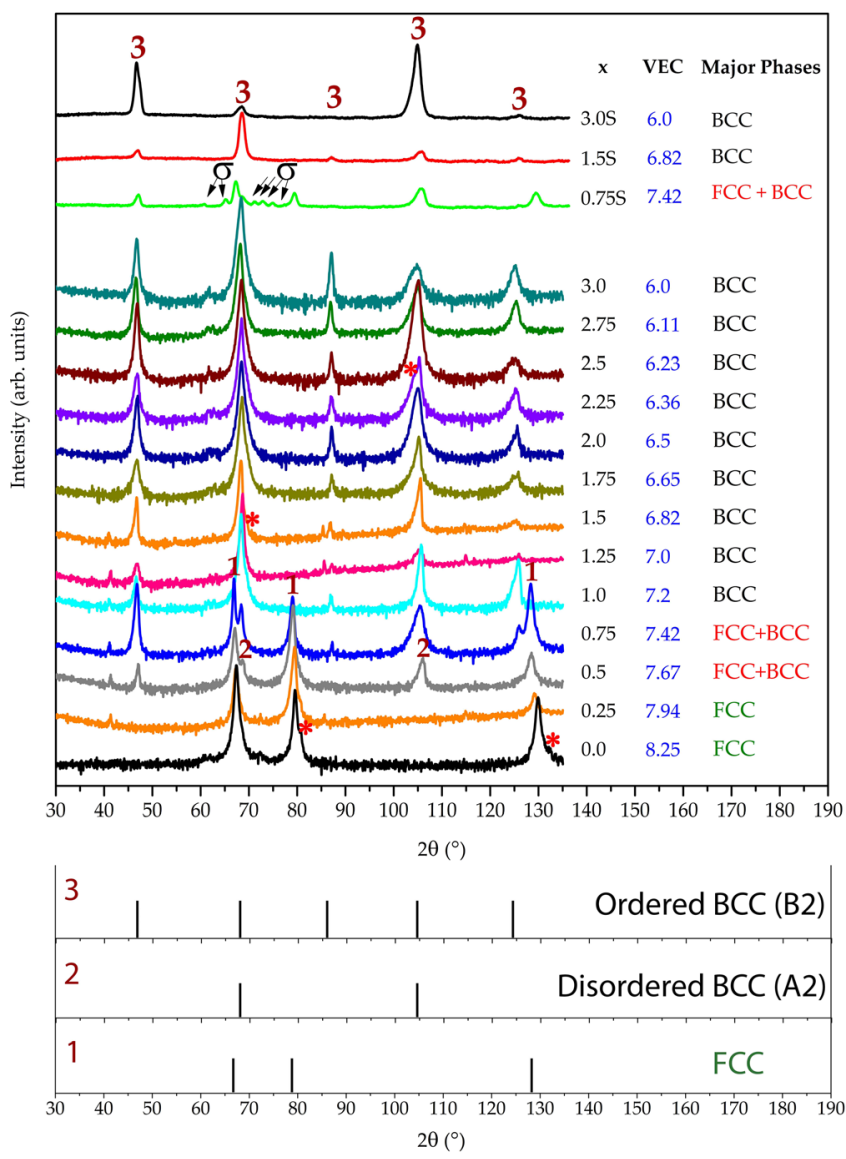

FIG. 1. Results from $\mathrm{x}$-ray diffraction data for $0.0 \leq x \leq 3.0$ on polished ascast samples and samples after Seebeck measurements at $900^{\circ} \mathrm{C}(x S)$ are presented together with their corresponding VEC value. The data are shown using a logarithmic $y$-scale in order to enhance minor features that would otherwise not to be seen on a linear $y$-scale. The different phases are marked as $1=\mathrm{FCC}, 2=\mathrm{BCC}$, and $3=\mathrm{B} 2$, while $\sigma$-phases are marked with arrows and $\sigma$. Red asterisks (*) mark the phases close in composition. 
amounts of Al, with $x=0.5$ and 0.75 (7.67 $\geq \operatorname{VEC} \geq 7.42)$, additional phases are present. These include disordered (A2) and ordered (B2) BCC type phases. Compositions in the range $1.0 \leq x \leq 3.0(7.2 \geq \mathrm{VEC} \geq 6)$ are observed to form a mixture of $\mathrm{A} 2$ and $\mathrm{B} 2$ phases. For $\mathrm{Al}_{0.75} \mathrm{CoCrFeNi}$, a (re)measurement after exposure to $T \leq 900^{\circ} \mathrm{C}$ during electrical resistivity and Seebeck coefficient measurements $(0.75 \mathrm{~S}$ in Figure 1) indicates the formation of minor intermetallic phases $(\sigma$-phase). Due to the strong preferred orientation (seen in the XRD data as strong variations in the relative intensity between peaks) commonly observed for HEAs, ${ }^{36}$ the determination of the actual weight fractions of different phases from XRD is subject to large errors, and it is therefore not presented in this study.

\section{Thermal analysis}

From the DSC measurements on samples with $0.0<x$ $\leq 3.0$ (see Figure 2), a background typical for metals is observed for all samples during heating. This change in background is related to irreversible changes in the material. For $x=0.25$ and 0.5 , no observable anomaly in the heat flow is observed. For $0.75 \leq x \leq 1.5$ (mixed FCC $+\mathrm{BCC}$ region), discernable endothermic features, however, start to appear in the temperature range $500^{\circ} \mathrm{C}<T<700{ }^{\circ} \mathrm{C}$, with a maximum in magnitude observed for $x=1.0$. At higher $\mathrm{Al}$ substitutions, i.e., $x>1$. 5, no significant features are observed. Finally, for $x=0.25$ (FCC) as well as $x=1.75,2.0,2.5,2.75$, and 3.0 (ordered BCC phases) smooth curves are observed up to $900^{\circ} \mathrm{C}$, indicating the absence of precipitation of secondary intermetallic phases.

\section{Electrical conductivity}

The average electrical conductivity for FCC, BCC + FCC, and BCC related phases are shown in Figure 3. We observed that the samples in this system are prone to the

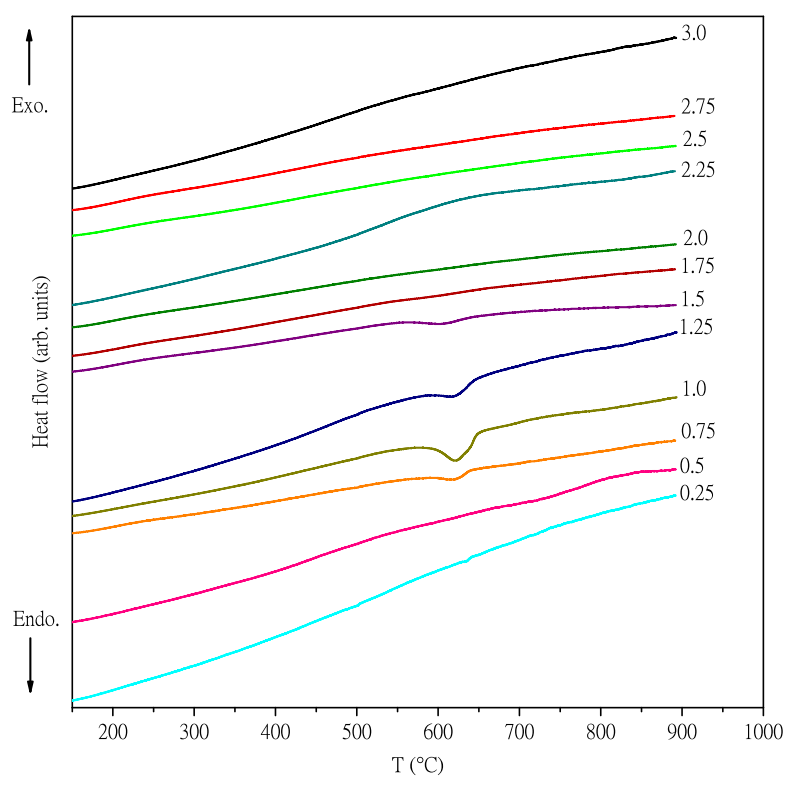

FIG. 2. Representative DSC curves of the samples with $0.0<x \leq 3.0$ measured from room temperature to $900{ }^{\circ} \mathrm{C}$ with a heating rate of $10^{\circ} \mathrm{C} \mathrm{min}{ }^{-1}$ under flowing Argon.

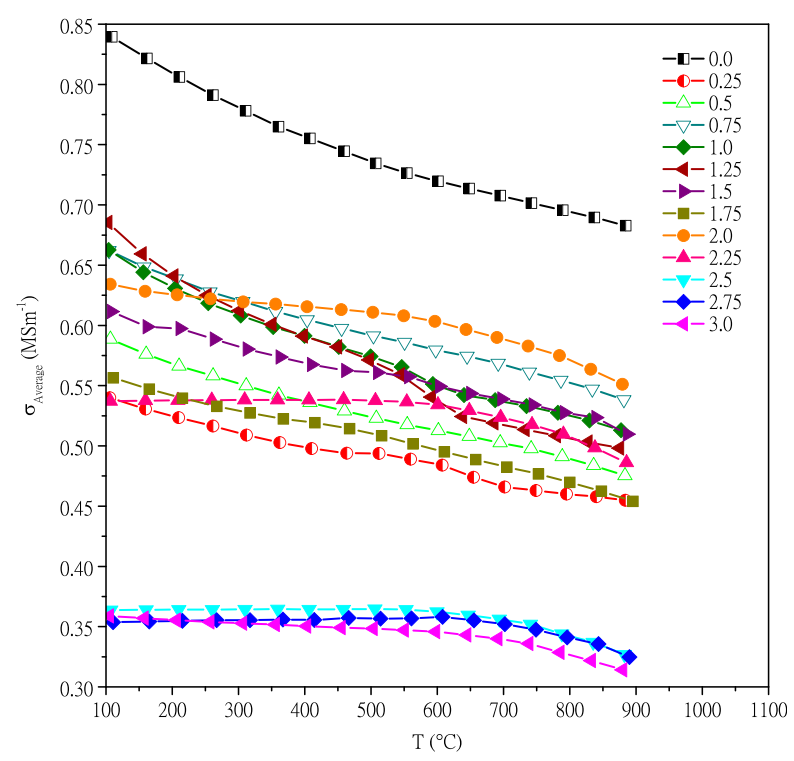

FIG. 3. Average electrical conductivity results for $0.0 \leq x \leq 3.0$ as a function of temperature.

formation of a thin surface layer that can affect the electrical conductivity values to a slight extent $(\sim 5 \%-15 \%)$. As a result, they do not follow an entirely systematic behavior between compositions close in $\mathrm{Al}$ content. The situation is further complicated by phase transformations (observed through DSC) involving the formation of intermetallic phases that change the matrix composition from its nominal value (see Figure 2). It is observed that $x=0.0$ shows the highest conductivity among not only the FCC phases but all the investigated compositions in this study. Among the FCC phases, the decrease in the electrical conductivity is followed by $x=0.5$ and 0.25 . In the $\mathrm{FCC}+\mathrm{BCC}$ region, the electrical conductivity for $\mathrm{Al}$ concentrations $0.75 \leq x \leq 1.25$ follows closely both qualitatively and quantitatively.

At higher $\mathrm{Al}$ contents $(x>1.25)$, the electrical conductivity behavior becomes less systematic in the range $1.5 \leq x \leq 2.25$ (i.e., the electrical conductivity is varying significantly between some compositions close in $\mathrm{Al}$ content). Again, the observed variations are most probably due to inevitable changes in the matrix composition and surface during heating, as well as preferential enrichment of elements with low melting points and high chemical affinity for each other. For compositions with $x>2.25$, the electrical conductivity behavior follows a systematic trend of decreasing electrical conductivity with increasing amount of Al. It is also observed that the compositions with high $\mathrm{Al}$ contents $\geq 2.0$ display a remarkably constant conductivity from room temperature up to high temperatures $\sim 600-650{ }^{\circ} \mathrm{C}$.

\section{Seebeck coefficient}

The average Seebeck coefficient values for FCC, $\mathrm{BCC}+\mathrm{FCC}$, and $\mathrm{BCC}$ phases as a function of temperature are shown in Figure 4. It is observed that for samples with the FCC structure, the absolute value of the Seebeck coefficient increases with the increasing $\mathrm{Al}$ content for $\mathrm{x} \geq 0.25$. Most compositions appear to be $n$-type. Positive values are, 


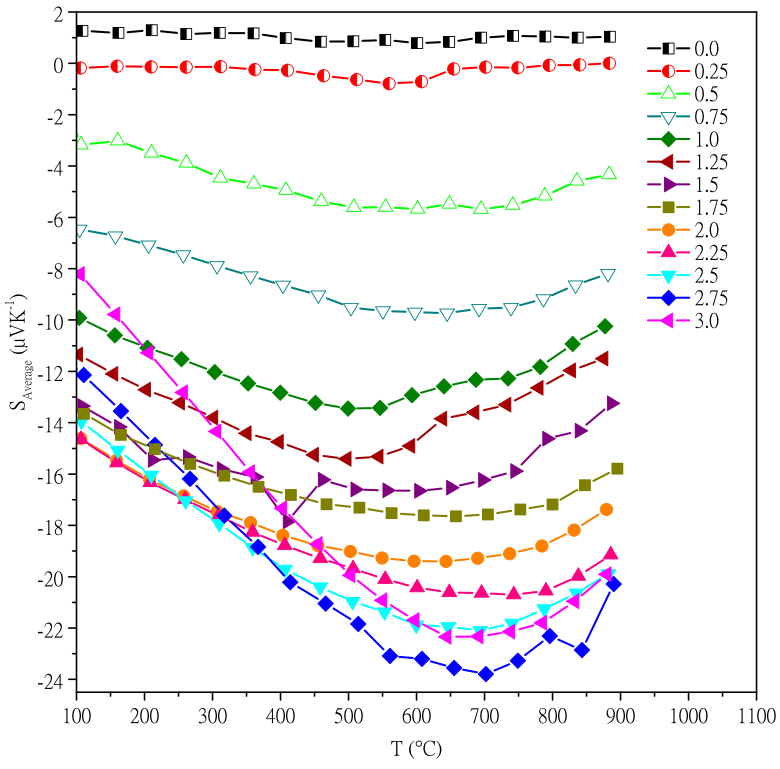

FIG. 4. Average Seebeck coefficients for $0.0 \leq x \leq 3.0$ as a function of temperature.

however, observed for $x=0.0$ at all measured temperatures, and in a smaller temperature range also for $x=0.25$. The trend of an increasing absolute value of the Seebeck coefficient continues with increasing $x$ throughout the FCC + $\mathrm{BCC}$ region, and the $\mathrm{BCC}$ region up to $x=2.75$ where a maximum value of $\sim-24 \mu \mathrm{V} \mathrm{K}^{-1}$ is reached for the Seebeck coefficient at $700^{\circ} \mathrm{C}$. Moreover, the absolute value of the Seebeck coefficients for this system increase over a wide temperature range from room temperature up to $900^{\circ} \mathrm{C}$ for $x \leq 2.5$, whereas for $x>2.5$, the temperature range around the maxima in the Seebeck coefficients becomes narrower and the slope becomes steeper below $600{ }^{\circ} \mathrm{C}$. Overall, a trend of an increasing absolute value for the Seebeck coefficient is thus observed, when the VEC is decreased (i.e., in the direction of higher $\mathrm{Al}$ content).

\section{Thermal conductivity}

Thermal conductivity was measured between $105-505^{\circ} \mathrm{C}$ for ingots with compositions $x=0.0,2.0,2.25,2.5,2.75$ and 3.0. Sample with $x=0.0$ was chosen as a reference point, while the samples with $x>1.75$ were selected for their lower electrical conductivities. In Figure $5, \kappa_{\text {tot }}$ for the measured samples is found to decrease significantly in comparison with the unsubstituted sample $(x=0.0)$. It is furthermore observed that $\kappa_{\text {tot }}$ decreases from $\sim 14.5 \mathrm{~W} \mathrm{~m}^{-1} \mathrm{~K}^{-1}$ for $x=0.0$ to $\sim 12.5 \mathrm{~W} \mathrm{~m}^{-1} \mathrm{~K}^{-1}$ for $x=2.25$ at $505^{\circ} \mathrm{C}$. In addition, $\kappa_{\text {tot }}$ seems to vary to some extent among different compositions at $505^{\circ} \mathrm{C}$, in contrast to the values at $105^{\circ} \mathrm{C}$, for which the $\kappa_{\text {tot }}$ values do not follow the initial order of $x=0.0,2.0,2.75$, 2.25, 2.5, and 3.0.

Due to interchanges during heating, the compositions end up in the order $x=0.0,2.75,2.0,2.5,3.0$, and 3.0 at $505^{\circ} \mathrm{C}$. These variations, although coming from small compositional and/or microstructural differences, fall within experimental errors of $\sim 3 \%-5 \%$ from the real values (see Figure S4, supplementary material). ${ }^{35}$

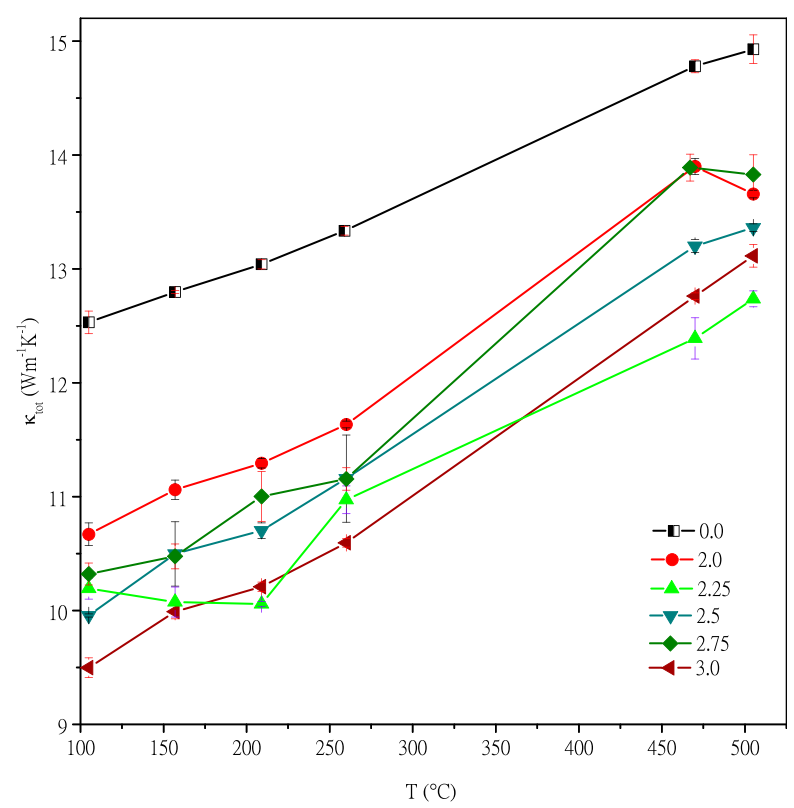

FIG. 5. Thermal conductivity values for samples with $x=0.0,2.0,2.25,2.5$, 2.75, and 3.0 between 105 and $505^{\circ} \mathrm{C}$. Errors within each measurement temperature for an average of 4 points are shown as error bars.

\section{DISCUSSION}

From the XRD and DSC data (see Figures 1 and 2, respectively), the connection between the formation of minor phases with different Al contents can be more clearly observed. In agreement with XRD data (see $0.75 \mathrm{~S}$ in Figure 1 ), which indicates the formation of a $\sigma$-phase after heat treatment during resistivity and Seebeck coefficient measurements, the DSC curves indicate a clear endothermic feature with an onset temperature at $\sim 580^{\circ} \mathrm{C}$ for $0.5 \leq x \leq 1.75$. These endothermic features have been reported as the formation of a $\sigma$-phase at around $600{ }^{\circ} \mathrm{C}$ and its dissolution around $\sim 930-960{ }^{\circ} \mathrm{C}$ depending on the $\mathrm{Al}$ content. ${ }^{37}$ Furthermore, XRD data agree well with DSC data for samples with $x \geq 1.75$ (i.e., no observable endothermic features at around $600^{\circ} \mathrm{C}$ ), hence indicating the absence of intermetallic secondary phases (e.g., 3.0 S in Figure 1). From the XRD patterns a shoulder is however sometimes observed for the main BCC peaks of $0.75<x<2.25$ (compare red asterisks in Figure 1). These shoulders can be attributed to, e.g., a coexisting disordered A2 phase with a composition close to the B2 phase. $^{38}$ Since similar features are also observed for $x=0.0$, they appear to originate from an FCC phase close in composition. In addition to the shoulders, it is observed that the main peaks for the BCC phases for $x>1.5$ gradually become broadened with increasing amount of Al. This broadening can be attributed to the reported spinodal decomposition of ordered $\mathrm{Al}$ and $\mathrm{Ni}$ rich $\mathrm{B} 2$ phases into $\mathrm{A} 2$ and $\mathrm{B} 2$ phases at $x>1.5 .^{37}$ The small crystallite sizes due to the spinodal decomposition as well as a broader distribution in the composition of the A2 and $\mathrm{B} 2$ phases certainly contribute to the broadening of the main peaks. In general, with increasing Al content, the peak positions are slightly shifted towards smaller angles $(2 \theta)$ corresponding to an increase in the average unit cell volume. This volume increase is attributed to the larger atomic radius of $\mathrm{Al}(\sim 1.4317 \AA)$ compared with 
the smaller transition metal atoms $(\sim 1.25 \AA) .{ }^{32}$ Finally, it can be assumed that the increasing $\mathrm{Al}$ content gradually increases the formation of local clusters of $(\mathrm{Co}, \mathrm{Ni}) \mathrm{Al}$ rich phases with ordered $\mathrm{B} 2$ structure (due to the large negative $\Delta \mathrm{H}_{\mathrm{Al}-\mathrm{Ni}}=-22 \mathrm{~kJ} \mathrm{~mol}^{-1}$ and $\Delta \mathrm{H}_{\mathrm{Al}-\mathrm{Co}}=-19 \mathrm{~kJ} \mathrm{~mol}^{-1}$ ), and will consequently contribute to the preference for the ordered $\mathrm{B} 2$ structure for high $\mathrm{Al}$ content.

From the electrical conductivity data, we observe that compositions with low $\mathrm{Al}$ content, starting from $x=0.0$, possess a high electrical conductivity $\left(\sim 0.85 \mathrm{MS} \mathrm{m}^{-1}\right)$. The electrical conductivity decreases (to $\sim 0.36 \mathrm{MS} \mathrm{m}^{-1}$ ) with increasing amounts of $\mathrm{Al}$ up to $x=3.0$ (see Figure 3). The observed trend is however not entirely systematic and shows small variations between different compositions (see Figure 3).

It is evident that the measurement technique and experimental sample preparation method influence the final electrical conductivity values to a certain extent $(\leq \sim 10 \%)$. For our investigated samples, we re-melted and re-casted some annealed samples and re-measured the properties, and no noticeable differences were observed in final results. In addition, we also tested samples cut from different directions of an ingot for Seebeck coefficient and electrical conductivity measurements. We found that after the 1st heating cycle all samples behaved in the same way (irrespective of which direction was used from the ingot), as the properties "equilibrated" during annealing. Thermal conductivity was, however, only measured for ingots. Re-melted samples were also tested, and they behaved in the same way as the original samples (see Figure S4). Comparison with values reported at $\sim 30, \sim 80$, and $\sim 130^{\circ} \mathrm{C}$ in Ref. 32 (see Figure 6) shows a strong variation of the values at the reported temperatures; these strong variations between our results and the reported values are, however, mainly in the FCC + BCC region. We attribute these variations to different experimental preparation methods and annealing procedures. This is further supported by the variations observed as a consequence of

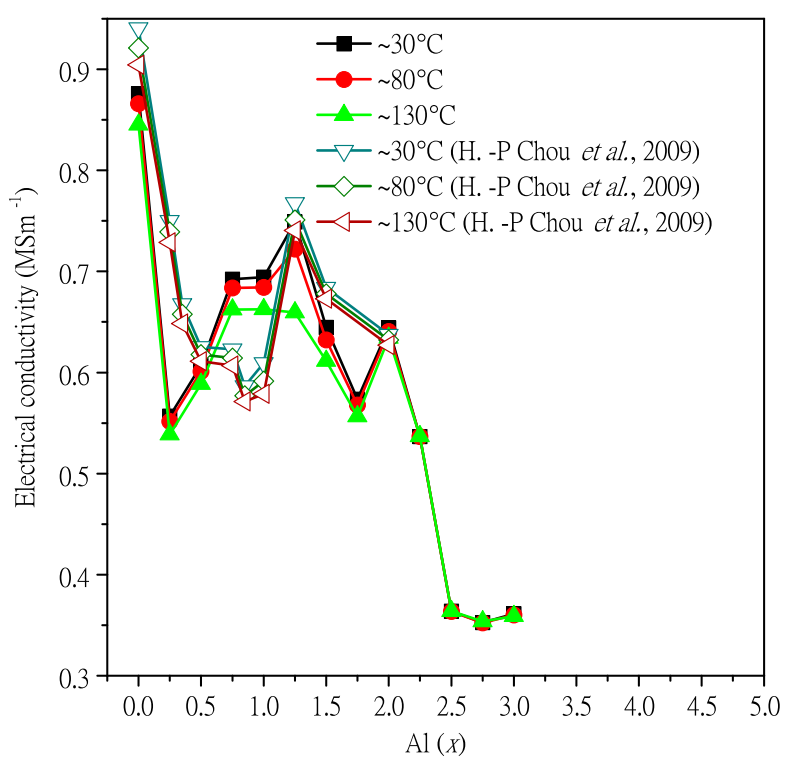

FIG. 6. A comparison between the electrical conductivity at $\sim 30,80$, and $130{ }^{\circ} \mathrm{C}$ from this study and Ref. 32 . different preparation methods (e.g., as-cast, annealing and quenching, and cold deformation by rolling), which can shift the electrical conductivity values by up to $\sim 30 \%$ for, e.g., $x=0.0 .{ }^{33}$ These variations in the measured values for the same composition can be compared with our results for, e.g., $x=0.0$, with electrical conductivity values changing between $\sim 0.86$ and $0.93 \mathrm{MS} \mathrm{m}^{-1}$ for two different measurements at room temperature (see Figure S1, supplementary material). ${ }^{35}$ Additionally, the surface of the alloys also changes slightly by the diffusion of different elements with lower melting points to the surface or a slight oxidation. This change in surface is observed as a slight coloring of the surface from a dark greenish color without $\mathrm{Al}(x=0.0)$ to slightly metallic pinkish color for intermediate $\mathrm{Al}$ content to increasing blueish/greenish color for high $\mathrm{Al}$ content $(0.0<x<2.5)$, and finally to a silver grey-blueish/greenish metallic color for the highest $\mathrm{Al}$ contents $(x>2.25)$. These color changes can mainly be attributed to the formation of different surface oxide layers ${ }^{39}$ and $\mathrm{Al}_{1-x} \mathrm{Ni}_{\mathrm{x}}$ alloys with varying $\mathrm{Al}$ contents, ${ }^{40}$ and also Al-free alloys judging by the dark green color of $x=0.0$. Furthermore, the diffusion of different elements in an HEA matrix has been reported to vary, and is correlated with the $\Delta \mathrm{H}_{\mathrm{A}-\mathrm{B}}$ values of the elements (i.e., relative differences in chemical interaction between different pairs of elements). ${ }^{12}$ The influence of the formation of different surface species cannot be entirely avoided during measurements due to occurring temperature dependent phase transitions (see Figures 1 and 2 for XRD and DSC data, respectively) at high temperatures, and could potentially affect the measured values. It is therefore worth noting that the electrical conductivity values can vary markedly for the same composition based on the preparation method/history ${ }^{33}$ and measurement conditions, and thus, will render the observed apparent variations of electrical conductivity values (see, e.g., $x=0.0$ and 1.75 in Figure S1 in the supplementary material). ${ }^{35}$ It is also worth mentioning that the darker oxide surface obtained upon annealing of the samples in air at $900{ }^{\circ} \mathrm{C}$ for $\sim 5 \mathrm{~h}$ could be easily polished by a fine $\mathrm{SiC}$ paper to restore the initial shiny metal surface. The effect from the formation of the surface oxide layer is something we noticed to have an impact; especially, this was noticeable if the contact was not polished well enough before electrical conductivity measurements in a physical property measurement system (PPMS) down to temperatures close to $4 \mathrm{~K}$ (not reported here). The trend and shape of the measurement results were similar, but the absolute value of the resistivity could vary with similar amounts as between $x=2.0$ and 1.75. After careful polishing, the electrical conductivity values from the PPMS corresponded well to the values obtained from the ZEM-3. For high temperature measurements, this effect is, however, much more difficult to assess, and also depends on the sample oxidation resistance.

The high electrical conductivity values that are expected as a result of an excessive number of charge carriers are believed to be a major contributor to the low absolute value of the Seebeck coefficient and the high thermal conductivity in the present system. A comparison of $\sigma$ and $S$ for different $x$ values can be made by grouping the compositions into $\mathrm{FCC}, \mathrm{FCC}+\mathrm{BCC}$, and $\mathrm{BCC}$ phases. In Figure 3, the 
conductivity curves for FCC phase samples are observed to decrease by $\sim 35 \%$ upon the introduction of $\mathrm{Al}(\sim 0.85 \mathrm{MS}$ $\mathrm{m}^{-1}$ for $x=0.0$ and $\sim 0.55 \mathrm{MS} \mathrm{m}^{-1}$ for $x=0.25$ ). The electrical conductivity values for $x=0.25$ and 0.5 are slightly different $\left(\sim 0.55\right.$ and $\sim 0.6 \mathrm{MS} \mathrm{m}^{-1}$ at room temperature, respectively), and can be ascribed to either the aforementioned effects from defects and/or to the formation of small amounts of secondary phases (minor BCC phases in $x=0.5) .{ }^{37} \mathrm{~A}$ small change in the composition of the main phase will consequently affect the value for the electrical conductivity. The Seebeck coefficient is, however, changing in a systematic order (following the increasing $\mathrm{Al}$ content) with an absolute value of $\sim 1 \mu \mathrm{V} \mathrm{K} \mathrm{K}^{-1}$ for $x=0.0\left(100^{\circ} \mathrm{C}\right.$ $\leq T \leq 900^{\circ} \mathrm{C}$ ), which increases to $\sim 5.5 \mu \mathrm{V} \mathrm{K} \mathrm{K}^{-1}$ for $x=0.5$ over the temperature range $400^{\circ} \mathrm{C} \leq T \leq 800^{\circ} \mathrm{C}$. The change in the Seebeck coefficient from positive (for $x=0.0$ ) to negative (see Figure 4) can at this point be ascribed to a change in the shape of the band structure close to the Fermi level due to the introduction of Al. Additionally, different scattering mechanisms may enhance the $n$-type conductivity in relation to the p-type conductivity. ${ }^{33}$ Furthermore, the decreasing ferromagnetic behavior with higher $\mathrm{Al}$ content is also indicative of an ordered (see Figure 1) Al-Ni rich phase. ${ }^{33}$ Such an ordered phase would, for Al contents below $44 \%$ ( $x=3.0$ corresponds to $\sim 43 \%$ ), be expected to show a negative Seebeck coefficient, very similar to what is observed in pure $\beta$-NiAl. ${ }^{17,40}$

Reported values of the Hall measurements ${ }^{33}$ from $x=0.0$ to 1.25 indicate a continuous decrease in the number of charge carriers with increasing $\mathrm{Al}$, while an increased carrier mobility is observed. These reported results indicate the important effect of $\mathrm{Al}$ on the charge carrier density and mobility in this system.

For higher Al contents $(0.5<x<1.5)$ within the FCC + $\mathrm{BCC}$ region the electrical conductivity in general decreases with increasing $x$. For these compositions it is, however, simultaneously observed (see Figure 4) that the absolute value of the Seebeck coefficients gradually increases (thus becoming increasingly more negative for larger $x$-values). This behavior is closely connected to the decreasing carrier concentration (in which a decrease would increase the absolute value of the Seebeck coefficient according to Equation (5)) reported from the Hall measurements for alloys with higher Al-contents in Ref. 33 and a possible increase in the mobility of $n$-type carriers in relation to $p$-type carriers. The Seebeck coefficient value can be expressed through the simplified relation,

$$
S=\frac{8 \pi^{2} k_{B}^{2}}{3 e h^{2}} m *\left(\frac{\pi \mu e}{3 \sigma}\right)^{2 / 3},
$$

where $n$ is the carrier concentration (where the mobility can be defined as $\mu=\sigma / e n$ ) and $m^{*}$ is the density-of-states effective mass of the carrier. ${ }^{1}$

In accordance with Equation (5), assuming that the total number of charge carriers decreases (with a factor of $\sim 1.9$ for $0.25 \leq x \leq 1.25)^{33}$ with increasing $x$, the mobility of the $n$-type carriers should be increased relative to the $p$-type carriers in order to cause an increase in the negative Seebeck coefficient. Additionally, the electrical conductivity for higher Al-content $(2.0 \leq x \leq 3.0)$ is found to systematically decrease with increasing $x$, while behaving nearly constant from room temperature up to $\sim 600^{\circ} \mathrm{C}$. For samples with $x=1.5$ and 1.75 the electrical conductivity deviates slightly from the trend and can be ascribed to small amounts of secondary phases that form during annealing, which appear as small features in the DSC data at higher temperatures $\sim 600^{\circ} \mathrm{C}$ (see Figure 2). These features are, however, not observed for higher Al-content. Overall, it is worth noting that the absolute value of the Seebeck coefficients at $T>650^{\circ} \mathrm{C}$ closely follows the trend of increasing $x$ (i.e., $|S|$ increases), although the maximum occurs for $x=2.75$ and not 3.0.

The formation of AlNi-rich domains as mentioned earlier can be expected to influence the overall Seebeck coefficient towards values closer to pure $\mathrm{AlNi}$, which at room temperature is $\sim 13-14 \mu \mathrm{V} \mathrm{K}^{-1}$ for $\mathrm{Al}_{0.4} \mathrm{Ni}_{0.6}$. This can be expected to occur for higher $x$-values, where the total $\mathrm{Al}$ content in the system reaches $\sim 43 \%$ and the probability of forming regions rich in $\mathrm{Al}$ and $\mathrm{Ni}$ is higher. ${ }^{17}$

Ideally, we can assume the conduction electrons to scatter with unpaired localized electrons (e.g., in magnetic atoms or ions) in narrow $d$-bands as in Kondo-like systems. ${ }^{41}$ In the $\mathrm{CoCrFeNi}$ system with strongly ferromagnetic atoms, lowering the VEC by the introduction of Al will inevitably, at some threshold concentration, create local clusters of magnetic transition metal atoms (i.e., Co and $\mathrm{Ni}$ ) surrounded by nonmagnetic Al, with substantial differences between dendritic (D) and interdendritic (ID) phases (see energy-dispersive $\mathrm{x}$-ray spectroscopy results of as-cast samples for $2 \leq x \leq 3$ in Refs. 37 and 42). For as-cast samples with compositions of, e.g., $x=2$, it is clearly observed that $\mathrm{Al}$ prefers to accumulate in either the $\mathrm{D}$ or the ID phase; however, with larger $x$-values the preference of $\mathrm{Al}$ seems less pronounced. For large $\mathrm{Al}$ concentrations (e.g., $x \geq 2$ ), the $d$-bands of the $3 d$-element clusters will effectively appear similar to defects, with the width of the $d$-bands varying with cluster size and degree of insulation by surrounding $\mathrm{Al}$ atoms. Thus, the formation of a local composite between magnetic and non-magnetic atoms for $x \geq 2.0$ (with ordered BCC structure) becomes significant enough for the appearance of the Kondo-effect at $\mathrm{T} \lesssim 280 \mathrm{~K}\left(\mathrm{~T}_{\mathrm{K}} \approx 90 \mathrm{~K}\right.$, $120 \mathrm{~K}$, and $280 \mathrm{~K}$ for $\mathrm{Fe}, \mathrm{Co}$, and $\mathrm{Ni}$, respectively). ${ }^{43}$ These clusters of transition metal atoms will, in these compositions, presumably interact with conduction electrons via $s p-d$ coupling. ${ }^{41,43}$ A Kondo-like effect has been reported for $x=2.0$ in these materials, ${ }^{33}$ and also in our previous findings for higher $x$ values $x>2.0$ we have observed a nearly constant resistivity behavior that can be related to the Kondo-like effect in this system.

In general, the conductivity in the system decreases from $\sim 0.85 \mathrm{MS} \mathrm{m}^{-1}$ to $0.36 \mathrm{MS} \mathrm{m}{ }^{-1}$ when moving from $x=0.0$ to $x=3.0$, hence indicating that the underlying electrical conductivity in the system can be changed using the simple VEC concept. For compositions where significant precipitation or phase transition is observed (indicated by DSC measurements), though the electrical conductivity values are affected and deviate from the trend. 
It is assumed from the lowering of the electrical conductivity that the electrical contribution to the thermal conductivity also follows a gradual decrease. This is observed from our thermal conductivity measurements, indicating a gradual reduction in the thermal conductivity from 12.5 to $9.5 \mathrm{~W}$ $\mathrm{m}^{-1} \mathrm{~K}^{-1}$ at room temperature. However, the order between different samples of varying $\mathrm{Al}$ content changes, and at $505^{\circ} \mathrm{C}$ the samples with $x=2.25$ and 3.0 achieve very similar thermal conductivities. The electrical contribution to the thermal conductivity in a metal can be estimated from the Wiedemann-Franz (W-F) law

$$
k_{e}=L \sigma T
$$

where $L$ is the Lorenz number $\left(2.44 \times 10^{-8} \mathrm{~W} \Omega \mathrm{K}^{-2}\right), \sigma$ is the electrical conductivity, and $T$ is the absolute temperature. The ratio of $k_{e} / k_{\text {latt }}$ (see Figure 7 ) shows that the electrical contribution to the thermal conductivity decreases gradually from $x=2.0$ to $x=3.0$ in relation to the initial value for $x=0.0$, which serves as the reference point. From the inset in Figure 7 , it is observed that the $k_{e} / k_{\text {latt }}$ ratio is close to unity for $x>2.25$. As the electrical conductivity values do not change significantly between the three samples $(x=2.5$, 2.75 , and 3.0), the electrical conductivity values most probably reach a minimum at $x>2.0$ for this particular system $(0 \leq x \leq 3)$.

In comparison with previously reported thermal conductivity values $^{32}$ for samples $x=0.0-2.0$, our system behaves slightly different. For the reported thermal conductivity values, ${ }^{32}$ BCC samples are responsible for the highest thermal conductivities in this system from room temperature up to $300{ }^{\circ} \mathrm{C}$, which is the maximum measurement temperature. Due to the complexity of multiprincipal element systems such as HEAs, differences in preparation might affect the final properties significantly.

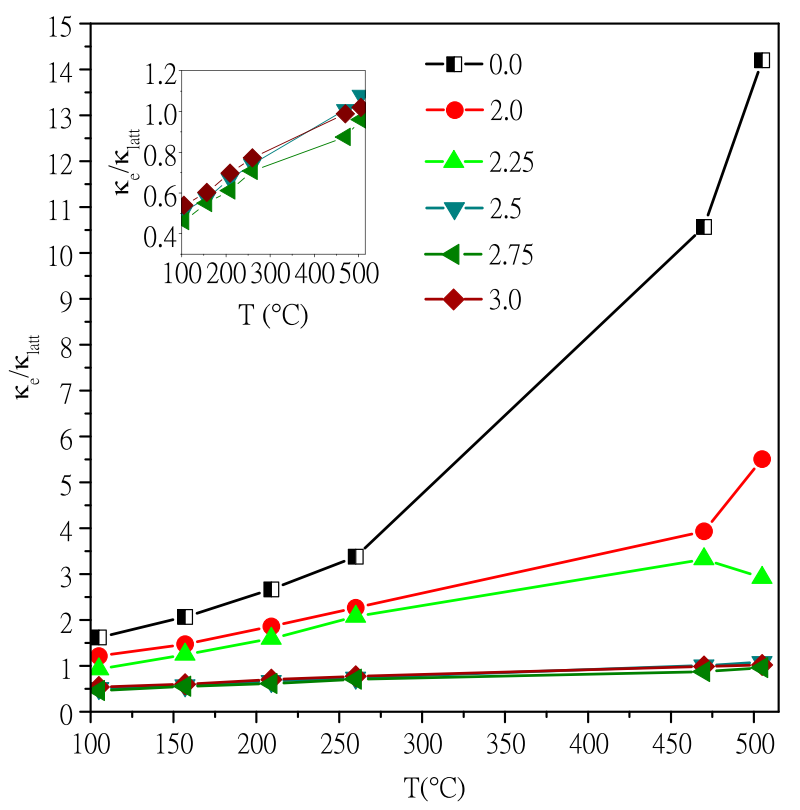

FIG. 7. Ratios between the calculated electrical and lattice thermal conductivity contributions are shown to illustrate the decrease in the electrical contribution with increasing $x$. For clarity, only samples with $x=2.5,2.75$, and 3.0 are shown in the inset.
We ascribe the difference in sample preparation to most of the differences observed (apart from the measurement technique). In Ref. 32, 40-50 g of material was used, which was melted and flipped at least 3 times, and furthermore, annealed for $24 \mathrm{~h}$ at $1100^{\circ} \mathrm{C}$, followed by water quenching. This preparation is different in comparison with our samples that were in total $\sim 60 \mathrm{~g}$ per ingot and flipped at least 5-6 times to ensure homogeneity. Samples that were cut out or cast as cylinders from pieces of the ingots were then annealed during the resistivity and Seebeck coefficient measurements from room temperature up to $900{ }^{\circ} \mathrm{C}$, and were cycled until no hysteresis effects were observed in resistivity and Seebeck coefficients $\left(\sim 5 \mathrm{~h}\right.$ total time at $\left.800-900^{\circ} \mathrm{C}\right)$. Moreover, the ingot samples in our study were melted and cooled directly on the water cooled copper plate and were annealed in a similar way as for the samples for resistivity and Seebeck coefficient measurements. As reported in Ref. 37 , the $\sigma$-phase already starts to form at $\sim 600^{\circ} \mathrm{C}$ and disappears at $\sim 930^{\circ} \mathrm{C}$ depending on $\mathrm{Al}$ content. The dissolution of the $\sigma$-phase above $\sim 930^{\circ} \mathrm{C}$ would therefore affect the phase constitution in the reported values from Ref. 32, in combination with the quenching procedure that freezes in the existing phases at the annealing temperature. Although $\sigma$-phases are highly electrically conductive phases, they will also act as phonon scattering centers, and can be assumed to affect the thermal conductivity values by lowering the phonon contribution $\left(\kappa_{\text {latt }}\right)$ and thus decrease $\kappa_{\text {tot }}$ (this can be assumed to happen in our samples where a maximum annealing temperature of $900{ }^{\circ} \mathrm{C}$ was used). It can be assumed that the highest thermal conductivity value for $x=1.5$ in Ref. 32 therefore can be related to the lowest $\sigma$-phase content in relation to our samples. This is partly reflected in the observed DSC curves for mainly $x=1.0-1.5$ (see Figure 2) and the large differences in electrical conductivity values for the same compositions (see Figure S1, supplementary material). ${ }^{35}$ In Ref. 32, it is reported that their samples are annealed at $1100{ }^{\circ} \mathrm{C}$ for $24 \mathrm{~h}$, which should be above the temperature for the decomposition of $\sigma$-phases before water quenching. This should produce much lower contribution from secondary phases, especially in the compositions where more than one phase is formed, e.g., $0.5<x<1.0$. In addition, their measurements only reached a maximum of $300^{\circ} \mathrm{C}$ for thermal conductivity and $\sim 100^{\circ} \mathrm{C}$ for electrical resistivity, which is most probably not sufficient to induce any major changes in this system. From Ref. 33, it is also clear that the microstructure affects the electrical resistivity significantly depending on preparation method. In Ref. 37, they investigate quite thoroughly the effect of temperature on different Al-contents for the reported system, and clearly there is a change in local composition and microstructure upon annealing at different temperatures.

From the power factors in Figure 8, it is observed that the highest absolute values of the Seebeck coefficients do not automatically render the best power factor (e.g., $x=2.75$ with $S_{\text {max }} \approx-24 \mu \mathrm{V} \mathrm{K}^{-1}$ at $T \approx 700^{\circ} \mathrm{C}$ ), but mainly the compositions with a reasonable combination of high Seebeck coefficients and high conductivity (e.g., $x=2.0$ and 2.75). In addition, one interesting feature of the investigated HEAs seems to be the broad range for which a high power factor is 


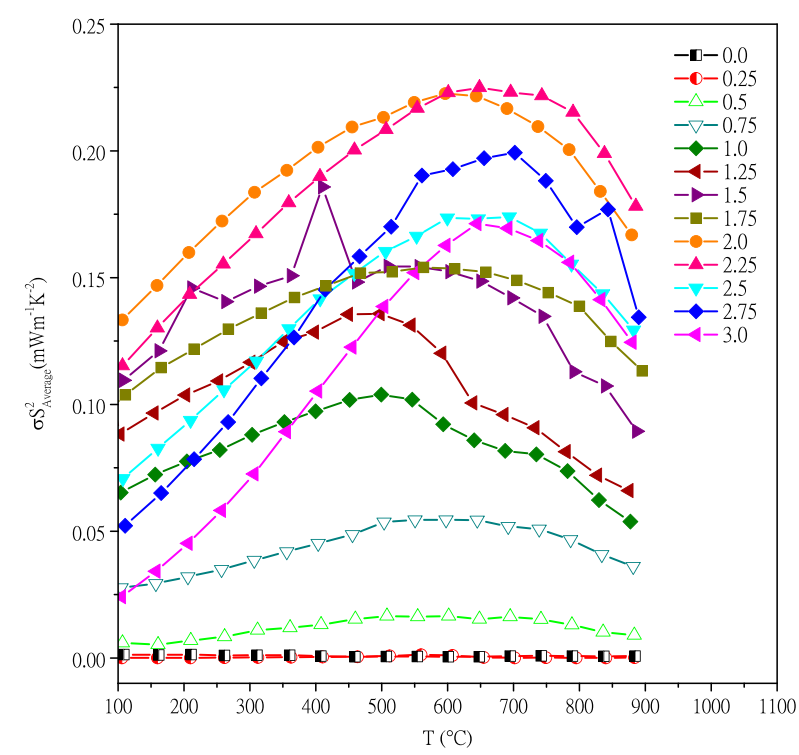

FIG. 8. Calculated power factors for all compositions. A maximum at $x=2.0$ and 2.25 is reached followed by samples with $x=2.75$ and 2.5 . It is worth noting the broad maxima of most samples in the HEA system.

maintained for most samples (e.g., $x>0.75)$. Most samples with higher $\mathrm{Al}$ content $(x \geq 2.0)$ seem to show a constant resistivity behavior, which is a desirable property for thermoelectric applications as this enables a larger operating temperature range. We believe that this constant resistivity property might arise from electron scattering from a complex microstructure (e.g., spinodal structure) and magnetic clusters. We are therefore currently trying to investigate it further, and results will be reported elsewhere. Furthermore, the $z T$ of the compositions are improved from $\sim 0$ for $x=0.0$ towards 0.015 for $x=2.0$ and 2.25 at $505^{\circ} \mathrm{C}$ (see Figure 9), thus indicating that higher $z T$ is achievable at higher temperatures close to the maxima between 600 and $800{ }^{\circ} \mathrm{C}$ for the Seebeck coefficient of the different compositions. The temperature range that is presented here as the optimum for this HEA system closes an important gap between half-Heusler

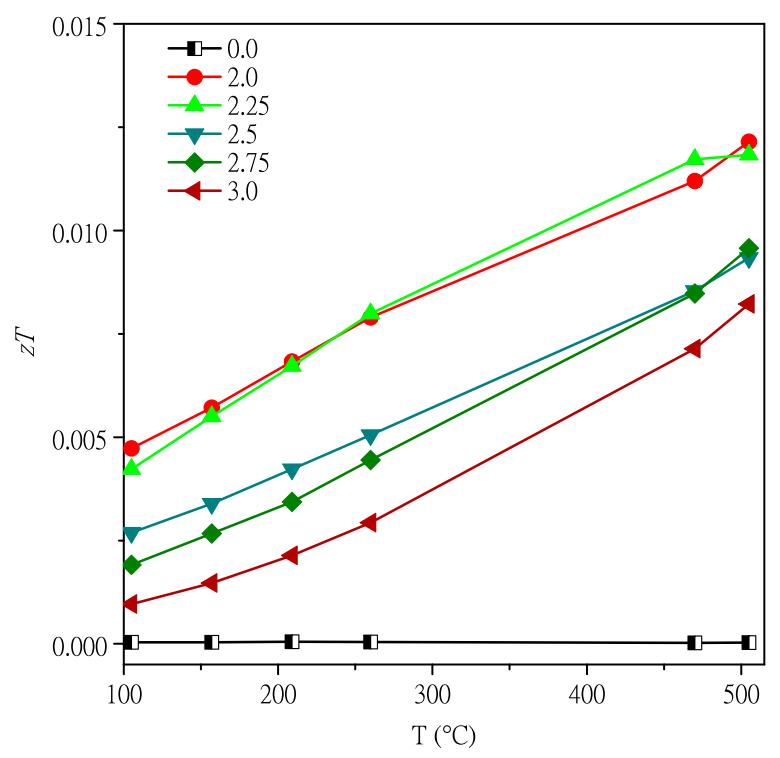

FIG. 9. The thermoelectric figure-of-merit $z T$ for the samples with $x>1.75$. compounds and most $n$-type materials $\left(T \approx 400-650^{\circ} \mathrm{C}\right)$ as well as $\mathrm{SiGe}$ type materials $\left(T \approx 850-1000^{\circ} \mathrm{C}\right)$ that work at temperatures below and above the investigated HEAs, respectively. The numerous possibilities of HEAs from a compositional point of view will open the door towards new high temperature TE materials. In comparison with halfHeusler alloys, HEAs allow microstructural and compositional design based on several well established parameters: (1) VEC, (2) atomic size differences, and (3) $\Delta \mathrm{H}_{\mathrm{f}}$ between different elements. Combining these three parameters, one can anticipate the design of high performance high temperature TE materials for future high temperature applications. Additionally, one would expect from the band structures of FCC and BCC that there should be a difference between the two structures regarding the Seebeck coefficient; however, the extent of this impact on the trends in a complex system as the presented one is not straightforward without too many simplifications. We therefore believe that it is necessary to combine both photoemission spectroscopy and inverse photoemission spectroscopy (from synchrotron sources) with band structure calculations, in order to understand the overall effects on the electronic properties from the changes in composition and structure.

From the results we conclude that the phonon contribution in these HEA materials can reach relatively low values of $\sim 3 \mathrm{~W} \mathrm{~m}^{-1} \mathrm{~K}^{-1}$ at $\mathrm{RT}$ for $x>2.25$ relative to alloys like, e.g., constantan $\left(\sim 5 \mathrm{~W} \mathrm{~m}{ }^{-1} \mathrm{~K}^{-1}\right)$. For the investigated HEA system, the $k_{\text {latt }}$ is already comparable with values achieved for common half-Heusler compounds. In comparison with other systems ${ }^{1}$ with some of the lowest $k_{\text {latt }}$ values for high temperatures, e.g., $\mathrm{La}_{3-\mathrm{x}} \mathrm{Te}_{4}\left(\sim 0.5 \mathrm{~W} \mathrm{~m}{ }^{-1} \mathrm{~K}^{-1}\right)$, clathrates $\left(\sim 1-2 \mathrm{~W} \mathrm{~m}^{-1} \mathrm{~K}^{-1}\right)$, and skutterudites $\left(\sim 1-3 \mathrm{~W} \mathrm{~m}^{-1} \mathrm{~K}^{-1}\right)$, the presented HEAs have still considerable room for improvement left. However, serious efforts should be directed towards maximizing and optimizing the Seebeck coefficients and the electrical conductivity in HEAs in order to make them applicable as high temperature TE materials. Especially a Seebeck coefficient of at least $100-200 \mu \mathrm{V} \mathrm{K}{ }^{-1}$ should be aimed for to reach reasonable performance. Future optimization of HEAs in regard to TE properties is indeed a challenge, which if successful, might lead to high performance alloys that are more compatible with $\mathrm{TE}$ generators than materials such as $\mathrm{SiGe}, \mathrm{Mg}_{2} \mathrm{Si}$, where good electrical contacts are often a concern. The potential to use refractory elements with high melting points in TE HEAs might also open the possibility to use TE materials for ultra-high temperature in demanding air/space applications. Additionally, there are substantial possibilities left for decreasing the lattice thermal conduction further (e.g., by adding heavier elements, by precipitating suitable intermetallic phases, or by using powder metallurgical routes to introduce additional microstructural complexity for phonon scattering). Furthermore, it is observed that there is a correlation between the electrical conductivity and the Seebeck coefficient with the overall VEC value of the system. This correlation opens the possibility to investigate a broad range of HEAs based on appropriate VEC values as starting points for good thermoelectric properties at high temperatures. 


\section{CONCLUSIONS}

High entropy alloys within the $\mathrm{Al}_{x} \mathrm{CoCrFeNi}$-system $(0.0 \leq x \leq 3.0)$ have been investigated for TE properties in the temperature range of $100-900^{\circ} \mathrm{C}$. We found that the addition of $\mathrm{Al}$ improves the $\mathrm{TE}$ properties through an increase in the maximum absolute value of the Seebeck coefficient ( $\sim 1 \mu \mathrm{V} \mathrm{K} \mathrm{K}^{-1}$ for $x=0.0$ to $\sim 23 \mu \mathrm{V} \mathrm{K} \mathrm{K}^{-1}$ for $x=3.0$ ), and a simultaneous decrease in the electrical conductivity (from $\sim 0.85 \mathrm{MS} \mathrm{m}^{-1}$ for $x=0.0$ to $\sim 0.36$ for $x=3.0$ ). The thermal conductivity is in addition decreasing from $\sim 15 \mathrm{~W}$

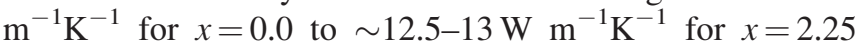
and 3.0, which is indicative of the lower electrical contribution $\left(k_{e}\right)$, to the total thermal conductivity. This is also reflected in the ratio between the electrical and lattice thermal conductivity $\left(k_{e} / k_{\text {latt }}\right)$, which decreases towards unity for high $\mathrm{Al}$ contents (i.e., $x=2.5,2.75$, and 3.0). Moreover, it is found that the investigated compositions reach a $z T \approx 0.015$ for $x=2.0$ and 2.25 at $\mathrm{T} \approx 505^{\circ} \mathrm{C}$, which was the upper limit for our thermal conductivity measurements. The $z T$ values follow the power factors for the different compositions with $x=0.0,2.0,2.25,2.5,2.75$, and 3.0. We therefore conclude that the potential to reach an intrinsically low lattice thermal conductivity through complexity in the microstructure for HEAs (e.g., by spinodal decompositions and mass fluctuation through substitutional elements), in combination with a systematic control of the electrical conductivity and the Seebeck coefficient through carefully chosen combinations of elements will open the possibility towards the design of high performance bulk TE materials for applications at high temperatures.

\section{ACKNOWLEDGMENTS}

The authors acknowledge the financial support from the Area of Advance Materials Science at Chalmers University of Technology. S.G. thanks the start-up funding from Area of Advance Materials Science at Chalmers. P.E. was partially supported by a Young Researcher Grant from the Swedish Research Council.

${ }^{1}$ G. J. Snyder and E. S. Toberer, Nat. Mater. 7, 105 (2008).

${ }^{2}$ M. S. Dresselhaus, G. Chen, M. Y. Tang, R. Yang, H. Lee, D. Wang, Z. Ren, J. P. Fleurial, and P. Gogna, Adv. Mater. 19, 1043 (2007).

${ }^{3}$ S. Chen and Z. Ren, Mater. Today 16, 387 (2013).

${ }^{4}$ J. de Boor, T. Dasgupta, H. Kolb, C. Compere, K. Kelm, and E. Mueller, Acta Mater. 77, 68 (2014).

${ }^{5}$ J. G. Noudem, S. Quetel-Weben, R. Retoux, G. Chevallier, and C. Estournès, Scr. Mater. 68, 949 (2013).

${ }^{6}$ C. J. Vineis, A. Shakouri, A. Majumdar, and M. G. Kanatzidis, Adv. Mater. 22, 3970 (2010).

${ }^{7}$ H. Xie, H. Wang, Y. Pei, C. Fu, X. Liu, G. J. Snyder, X. Zhao, and T. Zhu, Adv. Funct. Mater. 23, 5123 (2013).

${ }^{8}$ S. Chen, K. C. Lukas, W. Liu, C. P. Opeil, G. Chen, and Z. Ren, Adv. Energy Mater. 3, 1210 (2013).
${ }^{9}$ Q. Shen, L. Chen, T. Goto, T. Hirai, J. Yang, G. P. Meisner, and C. Uher, Appl. Phys. Lett. 79, 4165 (2001).

${ }^{10}$ S. Populoh, M. H. Aguirre, O. C. Brunko, K. Galazka, Y. Lu, and A. Weidenkaff, Scr. Mater. 66, 1073 (2012).

${ }^{11}$ Y. Zhang, T. T. Zuo, Z. Tang, M. C. Gao, K. a. Dahmen, P. K. Liaw, and Z. P. Lu, Prog. Mater. Sci. 61, 1 (2014).

${ }^{12}$ L. J. Santodonato, Y. Zhang, M. Feygenson, C. M. Parish, M. C. Gao, R. J. K. Weber, J. C. Neuefeind, Z. Tang, and P. K. Liaw, Nat. Commun. 6, 5964 (2015).

${ }^{13}$ O. N. Senkov, J. D. Miller, D. B. Miracle, and C. Woodward, Nat. Commun. 6, 6529 (2015).

${ }^{14}$ D. Miracle, J. Miller, O. Senkov, C. Woodward, M. Uchic, and J. Tiley, Entropy 16, 494 (2014).

${ }^{15}$ F. Zhang, C. Zhang, S. L. Chen, J. Zhu, W. S. Cao, and U. R. Kattner, CALPHAD: Comput. Coupling Phase Diagrams Thermochem. 45, 1 (2014).

${ }^{16}$ C. Tong, Y.-L. Chen, J. Yeh, S. Lin, S. Chen, T. Shun, C. Tsau, and S. Chang, Metall. Mater. Trans. A 36, 881 (2005).

${ }^{17}$ A. Manzoni, H. Daoud, R. Völkl, U. Glatzel, and N. Wanderka, Ultramicroscopy 132, 212 (2013).

${ }^{18}$ R. Valiev, Nat. Mater. 3, 511 (2004).

${ }^{19}$ L.-D. Zhao, V. P. Dravid, and M. G. Kanatzidis, Energy Environ. Sci. 7, 251 (2014).

${ }^{20}$ B. Gludovatz, A. Hohenwarter, D. Catoor, E. H. Chang, E. P. George, and R. O. Ritchie, Science 345, 1153 (2014).

${ }^{21}$ K. M. Youssef, A. J. Zaddach, C. Niu, D. L. Irving, and C. C. Koch, Mater. Res. Lett. 3, 95 (2015).

${ }^{22}$ M. J. Yao, K. G. Pradeep, C. C. Tasan, and D. Raabe, Scr. Mater. 72-73, 5 (2014).

${ }^{23}$ P. Koželj, S. Vrtnik, A. Jelen, S. Jazbec, Z. Jagličić, S. Maiti, M. Feuerbacher, W. Steurer, and J. Dolinšek, Phys. Rev. Lett. 113, 107001 (2014).

${ }^{24}$ Y. Zhang and W. J. Peng, Procedia Eng. 27, 1169 (2012).

${ }^{25}$ M.-H. Tsai, Entropy 15, 5338 (2013).

${ }^{26}$ G. A. Slack, in CRC Handbook Thermoelectrics, edited by D. M. Rowe (CRC Press, 1995), pp. 407-440.

${ }^{27}$ K. Biswas, J. He, I. D. Blum, C.-I. Wu, T. P. Hogan, D. N. Seidman, V. P. Dravid, and M. G. Kanatzidis, Nature 490, 570 (2012).

${ }^{28}$ Y. Pei, X. Shi, A. LaLonde, H. Wang, L. Chen, and G. J. Snyder, Nature 473, 66 (2011).

${ }^{29}$ S. Guo, C. Ng, J. Lu, and C. T. Liu, J. Appl. Phys. 109, 103505 (2011).

${ }^{30}$ S. Guo, Q. Hu, C. Ng, and C. T. Liu, Intermetallics 41, 96 (2013).

${ }^{31}$ F. R. de Boer, R. Boom, W. C. M. Mattens, A. R. Miedema, and A. K. Niessen, Cohesion in Metals: Transition Metal Alloys (Cohesion and Structure) (North Holland, 1989).

${ }^{32}$ H. P. Chou, Y. S. Chang, S. K. Chen, and J. W. Yeh, Mater. Sci. Eng., B 163, 184 (2009).

${ }^{33}$ Y.-F. F. Kao, S. K. Chen, T.-J. J. Chen, P.-C. C. Chu, J.-W. W. Yeh, and S.-J. J. Lin, J. Alloys Compd. 509, 1607 (2011).

${ }^{34}$ S. E. Gustafsson, Rev. Sci. Instrum. 62, 797 (1991).

${ }^{35}$ See supplementary material at http://dx.doi.org/10.1063/1.4935489 for additional material on thermoelectric properties.

${ }^{36}$ S. Guo, C. Ng, Z. Wang, and C. T. Liu, J. Alloys Compd. 583, 410 (2014).

${ }^{37}$ W. R. Wang, W. L. Wang, and J. W. Yeh, J. Alloys Compd. 589, 143 (2014).

${ }^{38}$ F. Findik, Mater. Des. 42, 131 (2012)

${ }^{39}$ D. König, C. Eberling, M. Kieschnick, S. Virtanen, and A. Ludwig, Adv. Eng. Mater. 17, 1365 (2015).

${ }^{40}$ H. Jacobi, B. Vassos, and H.-J. Engell, J. Phys. Chem. Solids 30, 1261 (1969).

${ }^{41}$ J. Kondo, Prog. Theor. Phys. 32, 37 (1964).

${ }^{42}$ C. Li, J. C. Li, M. Zhao, and Q. Jiang, J. Alloys Compd. 504, S515 (2010).

${ }^{43}$ M. R. Calvo, J. Fernández-Rossier, J. J. Palacios, D. Jacob, D. Natelson, and C. Untiedt, Nature 458, 1150 (2009). 